\title{
Computations of a Shock Layer Flow with a Vibrational-Specific Kinetics Model
}

\author{
M.-C. Druguet
}

Aix-Marseille Univ., CNRS UMR 7343, IUSTI, 5 rue Enrico Fermi, Marseille, 13453, France

\section{A. Bultel}

Normandie University, CNRS UMR 6614, CORIA, Site du Madrillet, St Etienne du Rouvray, 76801, France

V. Morel

Poitiers University, CNRS UPR 3346, PPRIME, ISAE-ENSMA, Futuroscope, 86962, France

J. Annaloro

CNES, DCT/TV/PR, BPI 1717, 18 Avenue Edouard Belin, Toulouse, 31401, France

Corresponding Author's name: marie-claude.druguet@univ-amu.fr

\begin{abstract}
The present work shows the ability of Navier-Stokes codes to handle detailed chemical kinetics models to compute the reactive and vibrational non-equilibrium gases in shock layers and the recent development done in our laboratories towards this goal. The detailed model used in the present study is a simplified version of a collisional-radiative model for $\mathrm{N}_{2}$, that has been implemented step-by-step in a Navier-Stokes code in order to show the effect of each group of chemical and vibrational processes. The processes taken into account are the vibration-vibration exchanges and the vibration-translation exchanges through molecular or atomic impact, leading to dissociation or not. The question of how the vibrational levels reach the Boltzmann distribution is also assessed. Comparisons of results obtained with global and detailed chemical kinetics models are performed.
\end{abstract}

\section{Introduction}

If simulating non-equilibrium gas flows in shock layers has been widely done so far with global models for the chemical reactions and the vibrational non-equilibrium relaxation, it is new to compute such flow fields with detailed chemical kinetics models. This is now possible as the CPU and memory resources of the computers allow running CFD codes with detailed models. State-specific vibrational models are efficient to model both the chemical reactions and the vibrational non-equilibrium at once, without making strong assumptions (like Boltzmann distribution) as it is the case with multi-temperature models for vibrational nonequilibrium modeling.

A major step has been done recently by IUSTI and CORIA in simulating the reactions between chemical species on their vibrational excited states (called pseudo species) and the evolution of these pseudo species in the shock layer surrounding a sphere flying into a nitrogen gas at a speed of $10.6 \mathrm{~km} / \mathrm{s}$, modeled by means of a simplified version of CoRaM-N a collisional-radiative (CR) model developed by CORIA [1]. This simplified model was recently implemented in the CFD code PINENS developed at IUSTI [2,3], in which the shock resolution is done with a shock-capturing method, an approximate Riemann solver in a finitevolume discretization of the Navier-Stokes equations. 


\section{State-to-State Modeling}

\subsection{Chemical Species and Vibrational Energy States}

The vibrationally specific model subject of the present work is a simplified version of the collisional-radiative model CoRaM-N 2 . It takes into account heavy, neutral particles only, as the CFD code in which it is implemented is not yet able to take into account electrons and ions. This model considers each of the 68 vibrational states of $\mathrm{N}_{2}(\mathrm{v}=0 \rightarrow 67)$ on the ground electronic state as pointed out by Armenise et al. [4], as well as $\mathrm{N}$ ground electronic state, that represent the 69 pseudo species whose population density evolutions are to be computed with a Navier-Stokes code.

\subsection{Collisional Elementary Processes}

According to the $\mathrm{CR}$ model CoRaM-N $\mathrm{N}_{2}$ that is used as the basis to derive the present vibrational-state specific model, the vibrational excitation of molecules $\mathrm{N}_{2}$ takes place through vibration-vibration processes under molecular impact (VV-m, 4489 processes), through vibration - translation processes under molecular impact (VT-m, 67 processes), or through vibration - translation processes under atomic impact (VT-a, 2125 processes). The data used for the rate coefficients for these processes were calculated by Esposito et al. [4] and Armenise et al. [5]. Once vibrationnally excited, the molecules $\mathrm{N}_{2}$ may dissociate during collisions. For the dissociation of $\mathrm{N}_{2}$ through vibration-vibration processes under molecular impact (VV-m-D, 67 processes), through vibration-translation processes under molecular impact (VT-m-D, 1 process), or through vibration-translation process under atomic impact (VT-a-D, 68 processes), the data of Esposito et al. [4] and of Armenise et al. [5] are also used. The total number of processes is 6817 forward reactions. The backward collisional elementary processes are calculated from the forward rate coefficient and the corresponding equilibrium constant using the detailed balance principle. Thus, the set represents a total of about 13,600 forward and backward elementary processes between 69 pseudo species.

\section{Results and Analysis}

Results obtained with Navier-Stokes simulations performed with the PINENS code are the evolution of $\mathrm{N}_{2}$ and $\mathrm{N}$ concentrations throughout the axi-symmetric shock layer past a 1meter radius sphere flying at $10.6 \mathrm{~km} / \mathrm{s}$, for the 68 vibrational states of $\mathrm{N}_{2}$ and the fundamental electronic state of $\mathrm{N}$.

\subsection{VV-m : 4489 Processes}

The first step in analyzing the effect of each group of processes on the flow field (temperature, species densities, ...) consists in considering the processes for the vibrationvibration exchanges by molecular impact (VV-m). Surprisingly, introducing the reactions corresponding to these processes does not produce any visible effect on the temperature nor on the species profiles in the shock layer. The shock position, the temperature, and the total density evolutions throughout the shock layer are the same when VV-m processes are taken into account as when no chemical processes are taken into account. Therefore, these processes are not taken into account in the following computations. 
The second step in analyzing the effect of a group of processes on the flow field and on the vibrational-level population densities consists in taking into account the vibrationtranslation exchanges by molecular impact (VT-m). Interestingly, the temperature profiles along the stagnation line (Fig. 1, left), are very similar to the results that are obtained when no chemical reactions are taken into account in the gas flow: the temperature is maximum in the entire shock layer, with a maximum reached near the stagnation area of the flow, right before the boundary layer. This means that the VT-m processes do not take much thermal energy from the gas. The evolutions of the mass fractions of each vibrational level of $\mathrm{N}_{2}$ (from $\mathrm{v}=0$ to 67) along the stagnation line throughout the shock layer, including the boundary layer, are also shown in Fig. 1, left. The fundamental vibrational level of $\mathrm{N}_{2}(\mathrm{v}=0)$ is depopulated across the shock wave to the profit of the excited states $v=1$ to 67 . As this is expected, the lowest levels are the most populated levels. The behavior of the first 10 vibrational levels right behind the shock wave is interesting: their population increases in the shock front, then decreases only $1 \mathrm{~mm}$ behind the shock front, to the profit of the excitation of higher vibrational states.
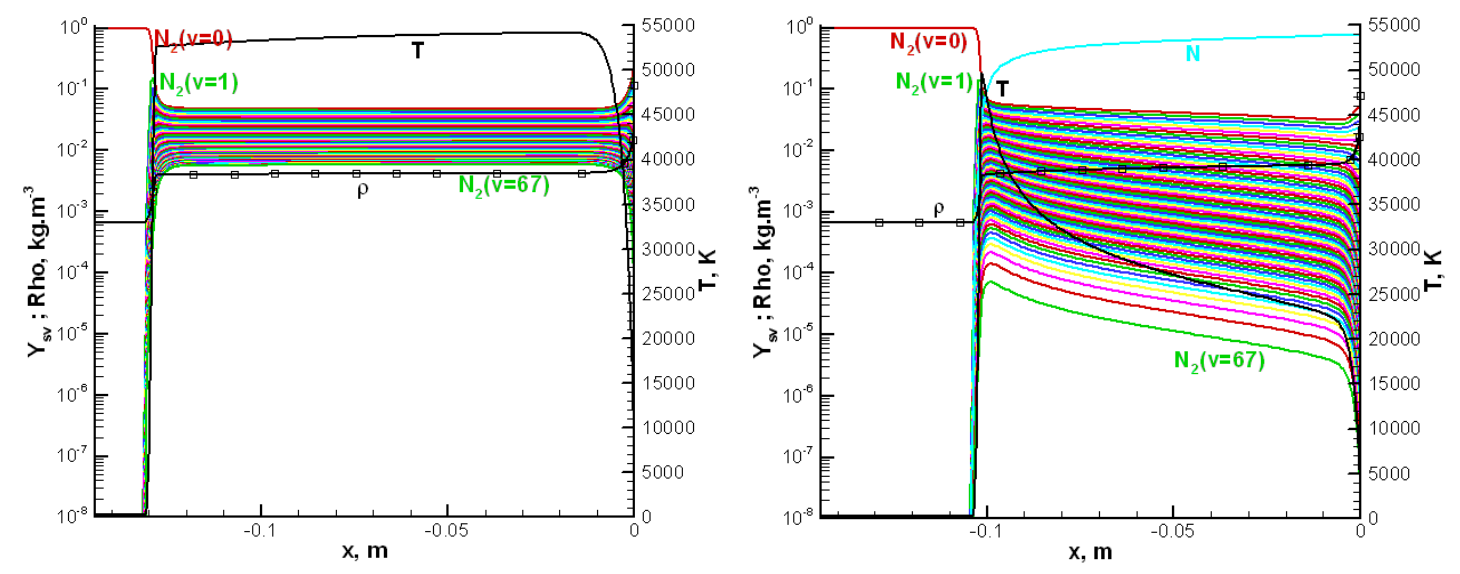

Fig. 1 Temperature and mass fractions of $\mathrm{N}_{2}(\mathrm{v}=0,67)$ and $\mathrm{N}$ along the stagnation line. Effect of the VT excitation by molecular impact. Left figure : processes VT-m are included in the computations ; Right figure : processes VT-m + VT-m-D are included

\subsection{VT-m + VT-m-D : 68 Processes}

The addition of the vibration-translation process by molecular impact leading to the dissociation (VT-m-D) leads to reduce the shock layer width from approximately $13 \mathrm{~cm}$ down to $10.5 \mathrm{~cm}$ (Fig. 1, right, compared to Fig.1, left). The introduction of this single process into the computations also changes the temperature profile across the shock layer: the temperature is no longer maximum at $53,000 \mathrm{~K}$ throughout the shock layer but peaks at $46,000 \mathrm{~K}$ right behind the shock front then decreases. This new temperature profile is similar to the temperature profiles usually observed when chemical reactions are taken into account through global models, and is typical of shock layer where dissociation processes take place. Regarding the species profiles within the shock layer, the atomic nitrogen is now the major species present in the shock layer. All the vibrational levels of $\mathrm{N}_{2}$ have been depopulated, especially the highest levels - because of the dissociation of $\mathrm{N}_{2}$ from the highest vibrational levels - to the profit of the atomic species. This means that the VT-exchange by molecular impact leading to dissociation (process VT-m-D) is a very efficient process among the many 
reactions of the vibrationally specific model implemented in the CFD code. This reaction of dissociation of $\mathrm{N}_{2}(\mathrm{v})$ also absorbs a large amount of thermal energy : the temperature that was approximately equal to $53000 \mathrm{~K}$ in the shock layer $2 \mathrm{~mm}$ from the stagnation point (see Fig. 1, left) is now equal to $22000 \mathrm{~K}$ at the same location (Fig. 1, right).

\subsection{VT-m + VT-m-D + VV-m-D : 135 Processes}

When the processes VV-m-D are added to the processes VT-m + VT-m-D in the simulations, the results are the same as when only the processes VT-m + VT-m-D are taken into account. This shows that the VV processes (leading to dissociation or not) are not efficient in the present test-case.

\subsection{VT-m + VT-m-D + VV-m-D + VT-a : 2260 Processes}

The introduction of the 2126 vibration - translation processes by atomic impact (VT-a) has a large effect on the flow field (Fig. 2, left, compared to Fig. 1, right): the reactions VT-a, like the process VT-m-D, lead to decreasing the shock layer width (from $10.5 \mathrm{~cm}$ to $8.5 \mathrm{~cm}$ approximately) and the temperature peak (from 46,000 K down to 38,000 K). Regarding the population densities in the shock layer, it is clear that the highest vibrational levels of $\mathrm{N}_{2}$ are even more depopulated to the profit of atomic nitrogen production than they were before. The curve of $\mathrm{N}$ population density (Fig. 2, left) shows that there is more $\mathrm{N}$ production in the shock layer when processes VT-a are considered, although there is no additional reaction of dissociation (only the VT-m-D reaction).
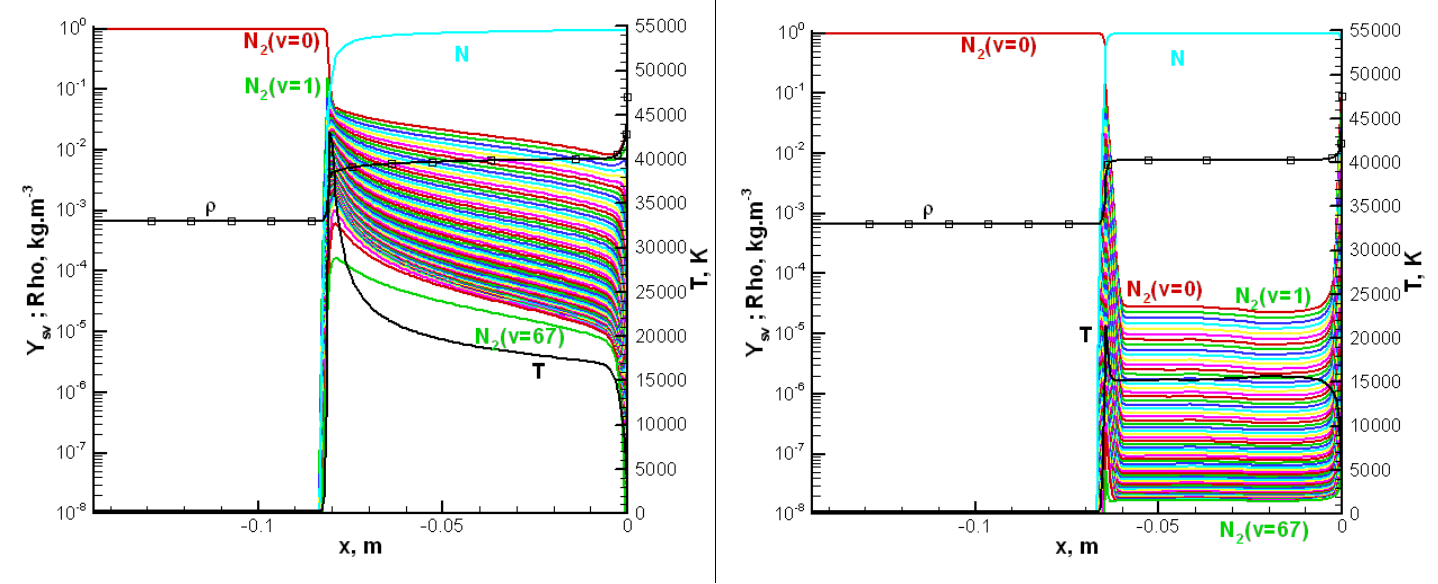

Fig. 2 Temperature and mass fractions of $\mathrm{N}_{2}(\mathrm{v}=0,67)$ and $\mathrm{N}$ along the stagnation line. Effect of the VT excitation by atomic impact. Left figure : processes VT-a are included in the computations in addition to the processes presented before ; Right figure : processes VT-a + VT-a-D are included in addition to the other processes

\subsection{VT-m + VT-m-D + VV-m-D + VT-a + VT-a-D : 2328 Processes}

Adding the 68 reactions corresponding to the vibration-translation processes by atomic impact leading to dissociation (VT-a-D) results in a complete dissociation of $\mathrm{N}_{2}$ (v) (see Fig. 2 , right). The flow field is strongly affected by the introduction of these additional reactions: again, the shock standoff distance is decreased (from $8.5 \mathrm{~cm}$ to $6.5 \mathrm{~cm}$ ) and the temperature 
peak is decreased from $38,000 \mathrm{~K}$ to $18,000 \mathrm{~K}$. Regarding the species densities, if the curves show that the various vibrational levels are all populated through the shock front, they are depopulated right behind the shock wave. This means that the additional reactions corresponding to the VT processes by atomic impact leading to dissociation (VT-a-D) are very efficient by transferring translational energy of atoms to vibrational energy of $\mathrm{N}_{2}(\mathrm{v})$ in order to dissociate the molecules, whatever their vibrational level. Interestingly, the temperature profile along the stagnation line slightly increases until the boundary layer where the temperature drops to reach to wall temperature.

\subsection{Is Boltzmann distribution reached in the shock layer?}

In order to show if the distribution of the vibrational level populations follows a Boltzmann distribution, Boltzmann plots of the $\mathrm{N}_{2}$ vibrational levels at different positions behind the shock front (marked 01 to 12) are presented in Figs 3, left and right. The Boltzmann distribution is reached when the curves representing the level populations (or mass fractions) - expressed in a logarithmic scale - versus the energies of the levels - expressed in a linear scale - are linear. The Boltzmann diagrams plotted in Figs. 3, left, show that the Boltzmann distribution is not reached for the case $(\mathrm{VT}-\mathrm{m})+(\mathrm{VT}-\mathrm{m}-\mathrm{D})+(\mathrm{VV}-\mathrm{m}-\mathrm{D})$. On the other hand, the Boltzmann distribution is reached after a distance of $5 \mathrm{~mm}$ behind the shock front in the case (VT-m) (not shown), $8.5 \mathrm{~mm}$ behind the shock front for the case (VT-m) + (VT-m-D) + (VV-m-D) (not shown), and $15 \mathrm{~mm}$ behind the shock front for the case (VT-m) + (VT-m-D) $+($ VV-m-D) + (VT-a) + (VT-a-D) (Fig. 3, right).
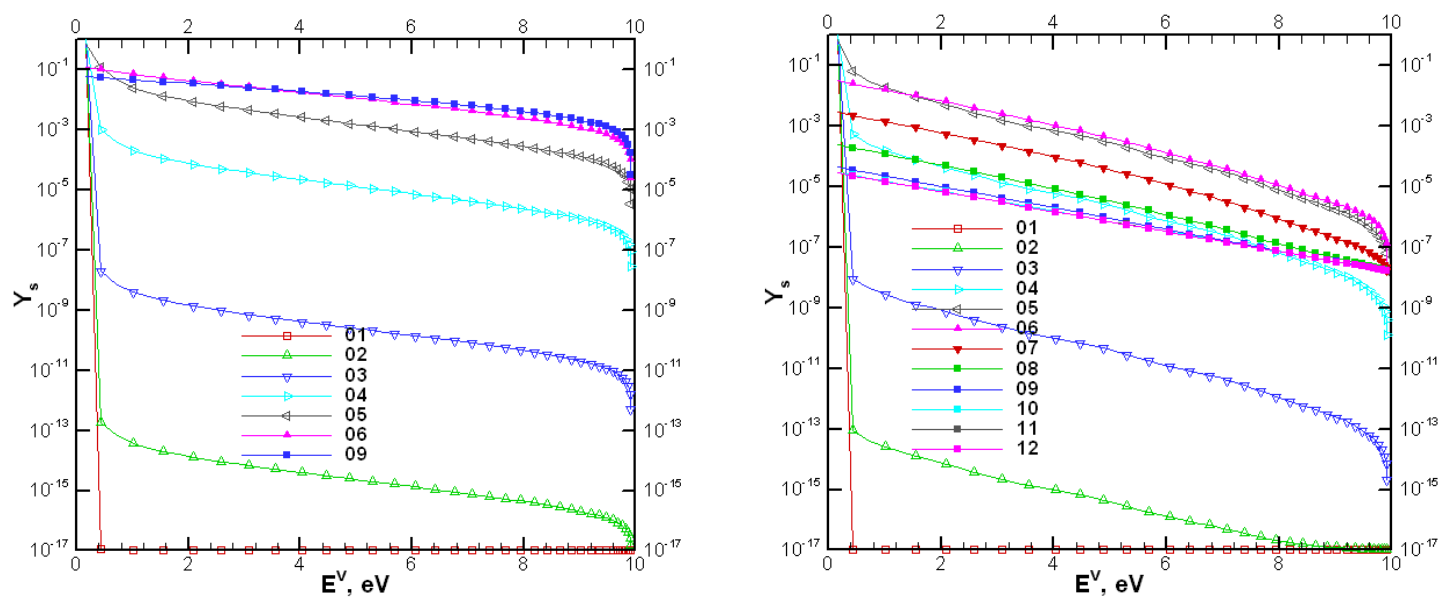

Fig. 3 Boltzmann plots across the shock wave. Left figure : processes (VT-m) +(VT-m-D) + $(\mathrm{VV}-\mathrm{m}-\mathrm{D})$ are included. Right figure : processes $(\mathrm{VT}-\mathrm{m})+(\mathrm{VT}-\mathrm{m}-\mathrm{D})+(\mathrm{VV}-\mathrm{m}-\mathrm{D})+(\mathrm{VT}-\mathrm{a})$ $+(\mathrm{VT}-\mathrm{a}-\mathrm{D})$ are included

3.8 Comparison of detailed and global kinetics rate models

Comparisons between results obtained with detailed models and results obtained with global models show how the global models perform compared to the detailed models in the prediction of the chemical species and vibrational states evolution past a shock wave (see Fig. 4). A reasonably good agreement is observed. 

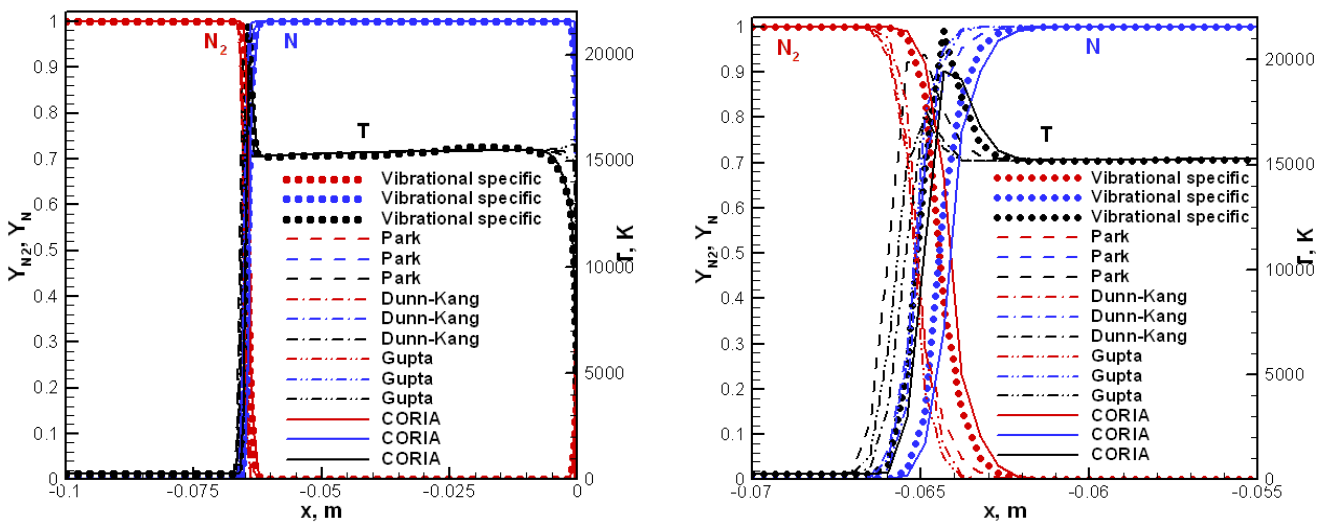

Fig. $4 \mathrm{~N}$ and $\mathrm{N}_{2}$ total mass fractions, and gas temperature along the stagnation line of the shock layer flow. Comparison between detailed (vibrational-specific, 2328 processes)) and global (Park, Dunn-Kang, Gupta, CORIA) chemical kinetics models. Right figure is a closeup view of the left figure

\section{Conclusion}

This work shows the ability of Navier-Stokes codes to handle detailed chemical models to compute the reactive and vibrational non-equilibrium gases in shock layers. The detailed model used in the present study has been implemented in the Navier-Stokes code step-by-step in order to show the effect of each group of chemical and vibrational processes in a given order. It was shown that the vibration-vibration exchanges do not have any visible effect on the present flow field, while the vibration-translation exchanges through molecular or atomic impact, leading to dissociation or not, are very efficient. Another main result is that the Boltzmann distribution of the vibrational levels is not reached until a certain distance behind the shock front. Finally comparisons between results obtained with global and detailed chemical kinetics models show good agreement. Future work is oriented towards the implementation of detailed models for Earth and Mars atmospheres into the PINENS code in order to simulate the non-equilibrium and reactive gas throughout an axi-symmetric shock layer with detailed models and compare the results with those obtained with global models classically used in such simulations.

\section{References}

[1] J. Annaloro, A. Bultel, P. Omaly, Collisional-radiative modeling behind shock waves in nitrogen, JTHT. 28, 4 (2014)

[2] R. Sanders, E. Morano, M.-C. Druguet. Multidimensional Dissipation for Upwind Schemes: Stability and Applications to Gas Dynamics. Journal of Computational Physics, 145, 2 (1998).

[3] M.-C. Druguet, G.V. Candler, I. Nompelis. Effect of Numerics on Navier-Stokes Computations of Steady Hypersonic Double-Cone Flows. AIAA Journal, 43, 3 (2005).

[4] F. Esposito, I. Armenise, M. Capitelli, $\mathrm{N}-\mathrm{N}_{2}$ state-to-state vibrational-relaxation and dissociation rates based on quasiclaisscal calculations, Chemical Physics. 331, 1 (2006)

[5] I. Armenise, F. Esposito, M. Capitelli, Dissociation-recombination models in hypersonic boundary layer flows, Chemical Physics. 336, 1 (2007) 